\title{
El rol del psicogastroenterólogo en los equipos de salud
}

\section{The psychogastroenterologist's role in the health teams}

\author{
Mariela González Tovar ${ }^{1}$
}

Citación: González T., M. (2021). El rol del psicogastroenterólogo en los equipos de salud. Psicología y Salud, 31(1), 123-129. https://doi.org/10.25009/pys.v31i1.2682.

\section{RESUMEN}

El objetivo del presente trabajo fue resaltar la importancia de la participación de los psicólogos en los equipos multidisciplinarios que atiendan a pacientes con enfermedades gastrointestinales. Existe una variedad de alteraciones emocionales que experimenta cada paciente, pero aun hoy se desconoce si son estas las que originan el padecimiento o son, por el contrario, las consecuencias del mismo, razón por la que es relevante atenderlas y valorarlas para poder tener una mejor comprensión de la enfermedad y, a la vez, lograr una mejor calidad de vida para los pacientes. Profundizar en estos aspectos ayudará a fundamentar una nueva especialidad de la psicología consagrada a estudiar los procesos relacionados con la aparición y el desarrollo de las enfermedades gastrointestinales, diseñar programas de promoción y prevención en el área, y apoyar el diagnóstico, la evaluación y el tratamiento médico-nutricional.

Palabras clave: Enfermedades gastrointestinales; Psicogastroenterología; Alteraciones emocionales; Equipos de trabajo.

\begin{abstract}
The present study aimed at emphasizing the importance of having psychologists in multidisciplinary health teams treating patients with gastrointestinal diseases. Although there is a variety of emotional alterations experienced by patients, there is no clear evidence on whether such alterations contribute to the origin of the disease or result as consequences of it. In this context it becomes important to assess and treat such alterations in order to better understand the disease and improve quality of life to these patients. In-depth analyses of these aspects would help define a new specialty of psychologsts dedicated to study psychological processes related to the onset and development of gastrointestinal diseases. This would in turn help design promotion and prevention programs in this area, and to support the diagnosis, evaluation, and medical-nutritional treatment.
\end{abstract}

Keywords: Gastrointestinal diseases; Psychogastroenterology; Emotional disturbances; Health teams.

\section{INTRODUCCIÓN}

$\mathrm{L}$

os trastornos funcionales gastrointestinales constituyen una parte importante de la patología gastroenterológica. Más de la mitad de los pacientes que acuden a consulta médica especializada lo hacen por este motivo, así como aproximadamente $5 \%$ de los que recurren a la atención primaria (Knowles, Monshat y Castle, 2013). Estas alteraciones son poco comprendidas por los médicos, quienes suelen confundirlos con trastornos psiquiátricos. Es así que los términos "funcional” y "psicosomático" se emplean

\footnotetext{
${ }^{1}$ Escuela de Psicología, Pontificia Universidad Católica de Chile, Av. Vicuña Mackenna 4860, Macul-Región Metropolitana, Santiago de Chile, Chile, correo electrónico: mgonzalez4@uc.cl. Artículo recibido el 10 de octubre de 2019 y aceptado el 28 de abril de 2020.
} 
como sinónimos, razón por la cual dichos desórdenes son mal diagnosticados o, peor aún, tratados inapropiadamente (Knowles et al., 2013; O'Sullivan, 2015).

Esos trastornos se definen como una combinación variable de síntomas gastrointestinales persistentes o recidivantes que no se explican por anomalías estructurales o metabólicas (Karantanos, Markoutsaki, Gazouli. Anagnou y Karamanolis, 2010; Longstreth et al., 2006). Incluso, la Clasificación Internacional de Enfermedades, en su décima revisión (CIE-10) (Organización Mundial de la Salud, 1992) los incluye bajo el epígrafe "Disfunción vegetativa somatomorfa" (F45.3), que comprende los trastornos del tracto gastrointestinal, sean enfermedades esofágicas, gastroduodenales o intestinales; entre las cuales se hallan las siguientes: síndrome de intestino irritable, reflujo gastroesofágico, enfermedad de Crohn, colitis ulcerosa, colecistitis, pancreatitis, dispepsia funcional y enfermedades inflamatorias del intestino (Podolski, 2002).

La fisiopatología de las citadas condiciones es extremadamente compleja, sobre todo al tratar de encontrar explicaciones para síntomas que al mismo tiempo no tienen un substrato orgánico evidente, como es el caso de la distensión abdominal, el estreñimiento, la diarrea, las náuseas y vómitos, la dificultad para deglutir, el sangrado al evacuar, la obstrucción intestinal y el aumento o la pérdida de peso repentinos (Podolski, 2002).

En algunos casos, según el diagnóstico, suelen ocurrir expresiones clínicas opuestas en un mismo paciente, por ejemplo la diarrea y el estreñimiento, lo que genera problemas para encontrar un tratamiento efectivo. Debido a ello, los mecanismos propuestos actualmente para comprender estas manifestaciones implican atender la ocurrencia de alteraciones en la sensibilidad visceral $\mathrm{y}$ en la motilidad intestinal ante ciertos factores psicosociales (Hall, 2016).

La hipersensibilidad visceral es la disminución del umbral doloroso, expresada como malestar o incomodidad abdominal. Para que exista, deben ocurrir alteraciones en la función de los diferentes niveles de procesamiento de la información sensitiva, es decir, tanto en el sistema nervioso entérico (SNE) como en la médula o en los centros más altos del sistema nervioso central (SNC) (Cranston, 2014; Karantanos et al., 2010).
Hoy día, la relación directa entre el SNC y el tubo digestivo se expresa a través del eje cerebro-intestino (gut-brain axis), desarrollando la idea de que eventos importantes que se originan en el SNC pueden interferir en la función del aparato digestivo (Carabotti, Scirocco, Maselli y Severi, 2015; Konturek, Brzozowski y Konturek, 2011). Siguiendo esta línea, en la investigación pionera de Fokudo Nomura y Muranaka (1993) se constata, por ejemplo, una respuesta exagerada del intestino al estrés. Esta relación del sistema digestivo y los estados de ánimo es lo que ha reforzado la existencia del eje cerebro-intestino y, en especial, de un "cerebro abdominal", al influir la serotonina en el estado de ánimo, así como también en el reflejo peristáltico.

Por otra parte, las alteraciones de la motilidad intestinal hacen referencia a cambios motores variados que hacen difícil un tratamiento efectivo. Por ejemplo, Hall (2016) señala que resulta complejo entender cómo algunos pacientes presentan una aceleración del tránsito del colon, expresado clínicamente como diarrea, mientras que otros manifiestan su disminución, esto es, estreñimiento o, peor aún, una alternancia de dichos síntomas.

La alteración motora más observada en los pacientes es la ocurrencia de una respuesta exacerbada del intestino en respuesta a varios estímulos, tales como la ingestión de alimentos, el estrés, la ansiedad y algunas drogas colinérgicas (Lee y Lee, 2016). Los mecanismos involucrados en esta hiperreactividad motora parecen estar relacionados con el funcionamiento de las interrelaciones entre el SNE y las fibras musculares lisas del tubo digestivo (Hall, 2016).

Gracias al conocimiento de cada una de estas respuestas se han mejorado los diagnósticos, más aún con la introducción de ciertos criterios que han permitido tener un mayor rigor en el diseño de estudios que pretenden identificar cada uno de los trastornos y la severidad de los cuadros (Lee y Lee, 2016), así como medir la eficacia terapéutica de medicamentos que buscan mejorar la calidad de vida del paciente que los padece (Shohi, Endo y Fukudo, 2018).

Teniendo en cuenta que cada una de estas enfermedades suele generar un conjunto de cambios de tipo personal, familiar, laboral y social (González, 2015; González y De Ascencao, 2005; Zas-Ros, 2016), y que los cambios en el estilo de 
vida de una persona se correlacionan con la incidencia ulterior de la enfermedad o la lesión y con la severidad de los síntomas (Holmes y Masuda, 1974), resulta importante entender los factores emocionales, ambientales y conductuales que participan en la aparición y modificación de las citadas enfermedades (Shohi et al., 2018).

Al ser una alteración biopsicosocial, en estos trastornos también participan factores cognoscitivos, emocionales, conductuales y fisiológicos (González y De Ascencao, 2005). Entre los factores cognoscitivos se incluyen el comportamiento enfermizo y las malas técnicas de adaptación a la enfermedad (Varona, Collado y Pinillos, 2009). Los emocionales se refieren a la presencia de algunos trastornos emocionales, como la ansiedad y la depresión (Bennebroek et al., 2011; Graff, Walker y Bernstein, 2009). Entre los conductuales se pueden señalar las situaciones estresantes o traumáticas de la historia comportamental del sujeto, mismas que pueden desencadenar el primer episodio de la enfermedad o producir la exacerbación de los síntomas (Vatn, 2009). Por último, el factor fisiológico comprende las alteraciones en la modulación del dolor, como la hipersensibilidad visceral que ocurre en algunos eventos fisiológicos gastrointestinales, así como también las alteraciones autonómicas que provocan alteraciones en la motilidad intestinal (Hall, 2016).

En conjunto, tales factores muestran que existe una alteración en la calidad de vida del paciente (González y De Ascencao, 2005; Tanimoto, Schmulson, Ramírez y Valdovinos, 1995), la que no depende únicamente de la severidad de los síntomas del trastorno (Hashem y El-Serag, 2003), sino también de la presencia de ciertas variables psicosociales (Huerta, Hinojoza, Santamaría y Schmulson, 2001).

\section{Abordaje psicológico de los trastornos funcionales gastrointestinales}

Cuando una persona padece un trastorno funcional gastrointestinal, cuya sintomatología se mantiene de forma persistente y afecta considerablemente su calidad de vida, comienza un largo recorrido de consultorios médicos especializados en gastroenterología, en los que recibe tratamientos que van aumentando en número, tornándose muchas de las veces irresoluble su situación.
Este hecho ha generado el interés por emprender una investigación sistemática y precisa de esas alteraciones desde una perspectiva bioconductual integrada, considerando la interacción entre eventos organísmicos y ambientales con significación psicológica (González, 2016). Se ha resaltado hasta ahora la manera en que los factores psicológicos pueden tener efectos en el curso de estas enfermedades; específicamente, el estrés percibido ha sido el más investigado al ser un factor de riesgo para padecerlas, pero también para exacerbar los síntomas que traen consigo (Daulabani, Veitía y Rodríguez, 2018; Maguen, Madden, Cohen, Bertenthal y Seal, 2014; Shah, Rezaie, Riddle y Pimentel, 2014).

Algunos estudios han mostrado un riesgo mayor de recaídas en pacientes con un elevado estrés percibido (Sajadinejad, Asgari, Molavi, Kalantari y Adibi, 2012; Shah et al., 2014), y asimismo muestran que $60 \%$ de los pacientes reportan sucesos negativos en su vida - especialmente divorcios o muertes de algún familiar-que marcan el inicio o la exacerbación de los síntomas (Daulabani et al., 2018; González, 2016). A la vez, estas situaciones estresantes suelen empeorar por los índices de ansiedad que sufren entre 40 y $60 \%$ de estos pacientes (Gros, Anthony, McCabe y Swinson, 2008; Roy-Byrne et al., 2008). También se ha observado que la comorbilidad psiquiátrica se asocia con un mayor riesgo de fracaso del tratamiento médico-nutricional, recaídas tempranas y, en algunos casos, la necesidad de cirugías (Crane, 2004; Daulabani et al., 2018; Dutta y Chacko, 2016; Micha1sen, Vandvik y Farup, 2015).

Ante esta situación, la psicología de la salud ha demostrado una considerable pluralidad de sus prácticas, ofreciendo apoyo no solo a los pacientes, sino también a los familiares y a los profesionales de la salud que trabajan en el equipo clínico, para el manejo de estrategias de afrontamiento de los cambios que se generan con la enfermedad (González, 2015; Zas-Ros, 2016). Igualmente, ha contribuido a entender la dirección en la cual los componentes emocionales y conductuales, junto con otros factores, participan en la aparición o modificación de las enfermedades gastrointestinales (Oblitas, 2005; Oblitas, 2006).

Por tanto, la inclusión de los psicólogos en las unidades multidisciplinarias resultaría fundamental para la atención integral de los pacientes 
con patologías gastrointestinales, contribuyendo así a mejorar las complicaciones, disminuir el impacto social y optimizar la calidad de vida del paciente (Daulabani et al., 2018; González, 2015; Johansson, Farup, Bracco y Vandvik, 2010).

Esta nueva disciplina de la psicología, llamada psicogastroenterología, aún no se ha constituido como una verdadera subespecialidad de la gastroenterología, tal como la psicooncología lo es de la oncología desde el año 1970 (Die Trie, 2004; Keefer, Palsson y Pandolfino, 2018). Desde hace varios años los especialistas que han estado trabajando con este tipo de pacientes han requerido su constitución para plantearla ya no solo de manera informal en consulta privada o durante las reuniones clínicas, sino de una manera más formal y profesional, para que de esa forma los nuevos avances en el campo de la gastroenterología contribuyan a mejorar no solo la atención paliativa de la sintomatología, sino también la calidad de vida de los pacientes y de quienes los rodean (González, 2015).

No obstante, recientemente la psicogastroenterología fue reconocida como un área de trabajo en varios artículos científicos del área de la gastroenterología cuando un grupo de médicos investigadores confirmaron que hay una desregulación del intestino-encéfalo que resulta crucial investigar y atender en consulta para deducir las condiciones gastrointestinales crónicas, y lograr así una terapia efectiva de las mismas (Keefer, 2018; Knowles, Keefer y Mikocka-Walus, 2019; Mikocka-Walus, Ford y Drossman, 2020). Queda esperar que las diferentes asociaciones y grupos médicos puedan incorporar en sus secciones, capítulos y equipos de atención a los psicogastroenterólogos como miembros en sus discusiones y estudios con la finalidad de aprovechar sus conocimientos y aportaciones, así como también para consolidar la psicogastroenterología como una disciplina por propio derecho, resaltando el rol que cada especialista tiene en el equipo de salud.

Con base en lo anterior, los trastornos funcionales gastrointestinales necesitan no solo de un abordaje médico con una tecnología avanzada y conocimientos terapéuticos de frontera, sino también de una visión holística de la persona, pues un elemento clave es un abordaje integral y la participación de un equipo multidisciplinario en defensa de una atención biopsicosocial, donde la psicogastroenterología puede desempeñar un importante papel (González, 2015).

Esta nueva subespecialidad, al estar interesada en la investigación de los factores psicológicos implicados en las enfermedades gastrointestinales y en los procedimientos de evaluación e intervención en estas, plantea que la psicoterapia y psicofármacos como los antidepresivos (subtipo de neuromoduladores centrales) contribuirían a encontrar una terapéutica efectiva para los referidos trastornos gastrointestinales, beneficiándose así tanto la salud psicológica como la gastrointestinal (Knowles et al., 2019; Mikocka-Walus et al., 2020).

Contar con un psicogastroenterólogo en el equipo multidisciplinario potenciaría el cuidado integral del paciente al profundizar en el conocimiento de los procesos psicológicos que afectan la aparición y adaptación a esas enfermedades (González, 2015; Keefer et al., 2018).

Al no esclarecerse la relación precisa entre los factores psicosociales y su repercusión en el campo de las enfermedades gastrointestinales, actualmente no se pueden plantear conclusiones definitivas sobre los factores específicos involucrados en su evolución (Quera y Palma, 2008), y aún menos sobre cuál es el rol del psicogastroenterólogo para hacer que sean efectivas las intervenciones destinadas a garantizar una mejoría en la calidad de vida del paciente que las padece.

Sobre esta base, se hizo necesario redactar el presente artículo, en el que no solo se reconoce el rol del psicogastroenterólogo en el campo clínico, sino también bosquejar las fases de su trabajo, teniendo como finalidad no únicamente identificar los factores que parecen influir en las enfermedades gastrointestinales y señalar los lineamientos a seguir para el diseño de programas integrales de intervención necesarios para mejorar la calidad de vida de estos pacientes.

A raíz de la falta de conocimientos sobre una terapéutica específica que sea efectiva para estos trastornos funcionales, se parte de lo señalado por Oblitas $(2005,2016)$ cuando menciona que el trabajo de un psicólogo de la salud comprende principalmente tres fases: a) prevención, b) intervención terapéutica y rehabilitación, y c) cuidados paliativos. Sin embargo, tal intervención pudiera diferir al atender pacientes con trastornos gastroin- 
testinales, pues dependerá del tiempo de diagnóstico de la enfermedad, de su evolución y del tratamiento sugerido por los especialistas médicos (Maguen et al., 2014).

A continuación, se muestra el desarrollo de estas posibles fases de atención, las cuales se basan en la experiencia personal de la presente autora en su práctica clínica como psicogastroenteróloga.

\section{Fase de diagnóstico}

Se caracteriza por ser un periodo de extrema incertidumbre para el paciente y su familia.

La etiología de las enfermedades gastrointestinales es aún materia de investigación al ser consideradas alteraciones biopsicosociales (González, 2015; Keefer, 2018; Maguen et al., 2014). Es por ello que algunas investigaciones concluyen que el desencadenamiento y el agravamiento de la elevada motilidad gastrointestinal pueden explicarse como factores emocionales debidos a dietas, fármacos, variaciones en la actividad hormonal y otros (Burke, Boumitri y Ananthakrishnan, 2017; González y De Ascencao, 2005; Whitehead y Schuster, 1985), lo que hace que en muchos casos el paciente tenga una larga historia de consultas médicas y de tratamientos para atender una enfermedad que ha alterado su calidad de vida, generando trastornos en sus relaciones sociales, trabajo, entretenimiento y actividad sexual (Daulabani et al., 2018).

En esta etapa se presentan diferentes alteraciones emocionales, especialmente ansiedad y depresión, lo que subraya la asociación detallada en la literatura entre estas alteraciones y los trastornos digestivos, detectada entre 40 y $60 \%$ de los pacientes diagnosticados (Daulabani et al., 2018; González, 2016; Maguen et al., 2014; Roy-Byrne et al., 2008; Shoji, Endo y Fukudo, 2018).

Estas alteraciones emocionales pueden observarse cuando el paciente, su familia, amigos y equipo de salud tratan de comprender la etiología multifactorial y la naturaleza funcional del padecimiento (González, 2016; Maguen et al., 2014). La intervención del psicogastroenterólogo en esta fase consiste en atender las necesidades emocionales, psicológicas y sociales que puedan tener el paciente y su familia con el propósito de guiarlos y disminuir así el malestar, y ofrecer apoyo emocio- nal para facilitar la percepción de control sobre la enfermedad, ayudándolos a entender que, si bien la enfermedad es de naturaleza funcional, aún hay estrategias de afrontamiento que se pueden utilizar para afrontarla.

\section{Fase de tratamiento}

La calidad de vida del paciente se compromete según sea la enfermedad gastrointestinal que padece, dado que cada una tiene una terapéutica clínica que depende de su evolución y de las características individuales de cada uno, esto es, de factores emocionales, sociales y conductuales (González, 2016).

En la fase de tratamiento los pacientes pueden tener dificultades para adherirse al tratamiento, y por esta razón el psicogastroenterólogo debe ayudarlos a potenciar sus estrategias de afrontamiento para hacer posible su adaptación a la enfermedad (Crane, 2004); trabajar con la percepción de rechazo que estos sienten de su medio social ante la enfermedad gastrointestinal; acompañarlos en el proceso de reincorporación a las diferentes actividades e intereses de su vida cotidiana, y asimismo estudiar el manejo de las contingencias con las personas que los rodean, en especial ante las verbalizaciones de dolor o molestia, para de esa forma analizar los cambios que pueden experimentar en sus relaciones sociales y familiares (González, 2016; González, García y Fernández, 1992).

\section{Fase de control y recaída}

Durante el tratamiento el paciente puede llegar a adaptarse a su condición y percibir que tiene el control de sus emociones y comportamientos; no obstante, durante esta fase puede haber un aumento de los niveles de ansiedad, según sean sus padecimientos y la evolución de estos, lo que puede desencadenar dudas y temores.

Ante esto, el psicogastroenterólogo tiene que abrir un espacio en el cual los pacientes hablen de sus emociones, dudas y temores, lo que le hará posible abordar sus alteraciones cognitivas, emocionales, motivacionales y conductuales que muchas veces alteran la efectividad de los tratamientos médicos (González, 2015; González, 2016); abordar y tratar las alteraciones emocionales que puedan 
generarse, y trabajar en la adaptación al nuevo estado patológico si volviera aparecer, repasando para ello las estrategias aprendidas para promover una mejor actitud ante el padecimiento.

\section{Otras funciones del psicogastroenterólogo}

Como cualquier otro psicólogo de la salud, el psicogastroenterólogo tiene otras funciones (Oblitas, 2005). En este caso, debe participar en el diseño de programas de prevención y promoción de la salud para fomentar la psicoeducación en el área de las enfermedades gastrointestinales. Debe también incorporar a los familiares y amigos en la terapéutica del paciente mediante, por ejemplo, terapias familiares para facilitar la comprensión de lo que implican la enfermedad y el tratamiento, reforzar estrategias trabajadas con los pacientes y desmitificar las concepciones erradas asociadas con la enfermedad gastrointestinal. Por último, debe asistir al equipo de profesionales de la salud en la atención de los pacientes aportando estrategias relativas a cómo manejar y atender a los pacientes según las alteraciones emocionales que pudieran sufrir, su perfil de personalidad y demás, en aras de lograr que el tratamiento sea efectivo y eficaz, a la vez que manteniendo la motivación y el interés de los profesionales en cada uno de los casos.

Llevando a cabo cada una de estas tareas, el psicogastroenterólogo podrá fomentar y mejorar la calidad de vida de los pacientes que padecen una enfermedad gastrointestinal, así como también atender su contexto inmediato.

En conclusión, es relevante aprovechar el rol que puede desempeñar el psicólogo en el equipo de salud gastroenterológico, así como lo que la nueva área de la psicogastroenterología puede aportar en la atención paliativa de la sintomatología de las enfermedades gastrointestinales, pero también garantizar un abordaje integral, en defensa de una atención biopsicosocial (González, 2015; Shoji et al., 2018).

La psicogastroenterología debe de ser reconocida por los diferentes profesionales de la salud como una disciplina científica, lo que hará posible llevar a cabo investigaciones y formular planes de atención e intervención en los diferentes equipos médicos, consolidándose así como un área que privilegie el papel del psicólogo en los equipos multidisciplinarios.

\section{REFERENCIAS}

Bennebroek Evertsz', F., Thijssens, N., Stokkers, P., Grootenhuis, M., Bockting, C., Nieuwkerk, P. y Sprangers, M. (2011). Do inflammatory bowel disease patients with anxiety and depressive symptoms receive the care they need? Journal of Crohns y Colitis, 6(1), 68-76.

Burke, K., Boumitri, C. y Ananthakrishnan, A. (2017). Modifiable environmental factors in inflammatory bowel disease. Current Gastroenterology Reports, 19(5), 21-22.

Carabotti, M., Scirocco, A., Maselli, M.A. y Severi, C. (2015). The gut-brain axis: interactions between enteric microbiota, central and enteric nervous systems. Annals of Gastroenterology, 28(2), 203-209.

Crane, C. (2004). Social learning affective state and passive coping in irritable bowel syndrome and inflammatory bowel disease. General Hospital Psychiatry, 26(1), 50-58.

Daulabani, N., Veitía, G. y Rodríguez C., M. (2018). Relación de eventos estresantes con el inicio de la enfermedad inflamatoria intestinal en pacientes del Hospital Vargas de Caracas. Revista Gen, 72(4), 86-90.

Die Trie, M. (2004). Jimmie Holland. Psicooncología, 1(1), 184-186.

Dutta, A. y Chacko, A. (2016). Influence of environmental factors on the onset and course of inflammatory bowel disease. World Journal of Gastroenterology, 22(3), 1088-1100.

González, M. (2015). La psicogastroenterología: una disciplina con presente y futuro. Revista Gen, 69(2), 22.

González, M. (2016). Factores psicosociales asociados con la calidad de vida en las enfermedades inflamatorias del intestino. Revista Gen, 70(1), 28-40.

González, M. y De Ascencao, M. (2005). Síndrome de intestino irritable. Aspectos psicológicos. Revista de la Facultad de Medicina de la Universidad Central de Venezuela, 28(2), 139-145.

González, M., García, E. y Fernández, C. (1992). Intervención conductual en el síndrome de intestino irritable: dos estudios clínicos. Psicothema, 4(2), 513-530.

Graff, L.A., Walker, J.R. y Bernstein, C.N. (2009). Depression and anxiety in inflammatory bowel disease: a review of comorbidity and management. Inflammatory Bowel Diseases, 15, 1105-1118. 
Gros, D., Anthony, M., McCabe, R. y Swinson, R. (2008). Frequency and severity of the symptoms of irritable bowel syndrome across the anxiety disorders and depressions. Journal of Anxiety Disorders, 23, 290-296.

Hall, J.E. (2016). Tratado de Fisiología Médica (13ª ed.). Barcelona: Elsevier.

Hashem, M. y El-Serag, H. (2003). Impact of irritable bowel syndrome: prevalence and effect on health -related quality of life. Gastroenterological Disorders, 3(2), S3-S11.

Holmes, T. y Masuda, M. (1974). Life changes and illness susceptibility. En B. S. Dohrenwend y B. P. Dohrenwend (Eds.): Stressful life events: Their nature and effects (pp 45-72). New York: John Wiley.

Huerta, I., Hinojoza, C., Santamaría, A. y Schhmulson, M. (2001). Diferencias en la calidad de vida (CV) entre pacientes con síndrome de intestino irritable (SII) y la población mexicana, evaluadas mediante el SF-36. Revista de Gastroenterología de México, 66(2), 145-146.

Johansson, P.A., Farup, P.G., Bracco, A. y Vandvik, P.O. (2010). How does comorbidity affect cost of health care in patients with irritable bowel syndrome? A cohort study in general practice. BMC Gastroenterology, 17, 10-31. Doi: 10.1186/1471-230X$10-31$.

Karantanos, T., Markoutsaki, T., Gazouli, M., Anagnou, N.P, Karamanolis, D.G. (2010). Current insights in to the pathophysiology of irritable bowel syndrome. Gut Pathogens, 2(1), 1-8.

Keefer, L. (2018). Behavioural medicine and gastrointestinal disorders: the promise of positive psychology. Nature Reviews Gastroenterology \& Hepatology, 15, 378-386.

Knowles, S.R., Keefer, L. y Mikocka-Walus, A. (2019). Psychogastroenterology for adults: A handbook for mental health professionals. London: Routledge.

Knowles, S.R., Monshat, K. y Castle, D.J. (2013). The efficacy and methodological challenges of psychotherapy for adults with inflammatory bowel disease: a review. Inflammatory Bowel Diseases, 19, 2704-2715.

Konturek, P.C., Brzozowski, T., Konturek, S.J. (2011). Stress and the gut: pathophysiology, clinical consequences, diagnostic approach and treatment options. Journal of Physiology and Pharmacology, 62(6), 591-599.

Lee, K.N. y Lee, O.Y. (2016). The role of mast cells in irritable bowel syndrome. Gastroenterology: Research and Practice, 1, $1-11$.

Longstreth, G.F., Thompson, W.G., Chey, W.D., Houghton, L.A., Mearin, F. y Spiller, R.C. (2006). Functional bowel disorders. Gastroenterology, 130(5), 1480-1491.

Maguen, S., Madden, E., Cohen, B., Bertenthal, D. y Seal, K. (2014). Association of mental health problems with gastrointestional disorders in Iraq and Afghanistan veterans. Journal of Depression and Anxiety, 31, 160-165.

Michalsen, V.L., Vandvik, P. y Farup, P. (2015). Predictors of health-related quality of life in patients with irritable bowel syndrome. A cross-sectional study in Norway. Health and Quality of Life Outcomes, 13(113). Doi: 10.1186/s12955-015-0311-8.

Mikocka-Walus, A., Ford, A.C. y Drossman, D.A. (2020). Antidepressants in inflammatory bowel disease. Nature Reviews Gastroenterology \& Hepatology, 17, 184-192. Doi: 10.1038/s41575-019-0259-y.

Oblitas, L. (2005). Atlas de psicología de la salud. Bogotá: PSICOM.

Oblitas, L. (2006). Psicología de la salud y calidad de vida (2ª ed.). México: Thomson.

Organización Mundial de la Salud (1992). CIE-10. Trastornos mentales y del comportamiento. Décima Revisión de la Clasificación Internacional de las Enfermedades. Descripciones Clínicas y pautas para el diagnóstico. Ginebra: OMS.

O’Sullivan, S. (2015). Todo está en tu cabeza. Barcelona: Planeta.

Podolski, D. (2002). Inflammatory bowel disease. New England Journal of Medicine, 347(6), 417-429. Doi: 10.1056/ NEJMra020831.

Quera, R. y Palma, R. (2008). Enfermedades inflamatorias intestinales: colitis ulcerosa y enfermedad de Crohn. Revista Médica de la Clínica Las Condes, 19(4), 331-341.

Roy-Byrne, P., Davidson, K., Kessler, R., Asmundson, G., Goodwin, R., Kubzansky, L. et al. (2008). Anxiety disorders and comorbid medical illness. General Hospital Psychiatry, 30, 208-225.

Shah, E., Rezaie, A., Riddle, M. y Pimentel, M. (2014). Psychological disorders in gastrointestinal disease: epiphenomenon, cause or consequence? Annals of Gastroenterology: Quarterly Publication of the Hellenic Society of Gastroenterology, $27(3), 224-230$.

Shoji, T., Endo, Y. y Fukudo, S. (2018). Psycho-gastroenterology. En K. Tominaga y H. Kusunoki (Eds): Functional dyspepsia (pp. 105-115). Singapore: Springer.

Tanimoto, M., Schmulson, M., Ramírez, D. y Valdovinos, M. (1995). Prevalencia de trastornos funcionales digestivos en estudiantes de medicina. Revista de Gastroenterología de México, 60(3), 57.

Varona, L., Collado, E. y Pinillos, A. (2009). Terapia cognitivo-conductual en la enfermedad de Crohn. Norte de Salud Mental, $8(34), 117-123$.

Vatn, M.H. (2009). Natural history and complications of IBD. Current Gastroenterology Reports, 11(6), $481-487$.

Zas-Ros, B. (2016). Experiencias en psicología hospitalaria. Lima: ALFEPSI. 